\title{
Per-antenna Power Minimization in Symbol-Level Precoding
}

\author{
Danilo Spano*, Maha Alodeh*, Symeon Chatzinotas*, and Björn Ottersten* \\ ${ }^{*}$ SnT - securityandtrust.lu, University of Luxembourg \\ email: \{danilo.spano, maha.alodeh, symeon.chatzinotas, bjorn.ottersten\}@uni.lu
}

\begin{abstract}
This paper investigates the problem of the interference among multiple simultaneous transmissions in the downlink channel of a multi-antenna wireless system. A symbol-level precoding scheme is considered, where the data information is used, along with the channel state information, in order to exploit the multi-user interference and transform it into useful power at the receiver side. In this framework, it is important to consider the power limitations individually for each transmitting antenna, since a common practice in multi-antenna systems is the use of separate per-antenna amplifiers. Thus, herein the problem of per-antenna power minimization in symbol-level precoding is formulated and solved, under Quality-of-Service constraints. In the proposed approach, the precoding design is optimized in order to control the instantaneous power transmitted by the antennas, and more specifically to limit the power peaks, while guaranteeing some specific target signal-to-noise ratios at the receivers. Numerical results are presented to show the effectiveness of the proposed scheme, which outperforms the existing state of the art techniques in terms of reduction of the power peaks and of the peak-to-average power ratio across the transmitting antennas.
\end{abstract}

\section{INTRODUCTION}

During the last decade, there has been a strong growth in the demand for interactive services and multimedia content delivery through wireless networks. This ever-increasing demand imposes stringent throughput requirements for the next generation of communication systems. Consequently, a major challenge is to find feasible technical solutions to achieve this target, taking into account that the wireless spectrum is a scarce resource, which is getting more and more congested.

A way to tackle this challenge is to resort to multi-antenna transmitters, which allow to aggressively reuse the frequency spectrum by exploiting the additional degree of freedom given by the space dimension. This way, different users can be served by the transmitter sharing the same time and frequency resources, through a space division multiple access (SDMA) scheme [1]. Nonetheless, the full frequency reuse advantages come with the need of handling the interference arising between the simultaneous transmissions addressed to the co-channel users, which is the main limitation in these kind of systems. In this context, advanced signal processing techniques, such as linear precoding (or beamforming), have been shown to be an effective way to manage the multi-user interference (MUI), while guaranteeing some specific service requirements [2]-[7].
Precoding techniques can be classified in channel-level and symbol-level. The conventional approach is the channel-level one where, using the knowledge of the channel state information (CSI), the transmitted signals are precoded in order to mitigate the MUI. In this framework, different strategies have been considered for the precoder design. The optimal precoding strategy for the minimization of the total transmit power, whilst guaranteeing some Quality-of-Service (QoS) targets at each user was given in [5], [7], while the problem of precoding for maximizing the minimum signal-to-interferenceplus-noise ratio (SINR) across the users, under sum power constraints (SPC), was optimally solved in [6]. The goal of the latter formulation is to increase the fairness of the system, hence the approach is also referred to as max-min fair. This work on channel-level precoding was extended in [8] accounting for per-antenna power constraints (PAC), and in [9] considering generalized power constraints. Furthermore, the problem of channel-level precoding in a multigroup multicast framework has been tackled in [10].

On the other hand, in symbol-level precoding [11]-[18] the transmitted signals are designed based on the knowledge of both the CSI and the data information (DI), constituted by the symbols to be delivered to the users. In this approach, where the design exploits also the DI, the aim is not to eliminate the interference, but rather to control it so to have a constructive interference (CI) effect at each user. In [11] the classification of the interference as constructive or destructive was given, and a selective channel inversion scheme was proposed in order to eliminate the destructive interference. A more advanced symbol-level precoding scheme was proposed in [12], based on the rotation of the destructive interference, with the aim to transform it into useful power. Similarly to the channel-level case, also in this approach different optimization strategies have been considered in the literature. In [14] the sum power minimization and the max-min fair problem were solved for M-PSK modulations. Extensions of such works include optimization strategies for multi-level modulations [15] and more flexible approaches for exploiting the constructive interference [17]. Furthermore, symbol-level precoding has been considered also in relation to multicast-based systems [17], and taking into account the imperfect knowledge of the CSI [18]. However, the problem of limiting the per-antenna transmit power in the context of symbol-level precoding has not been addressed yet. 
In this work, the problem of constructive interference for per-antenna power minimization, under QoS constraints, is considered and solved for M-PSK modulations. The motivation for the proposed approach is twofold. A first consideration, valid for any precoding technique, is that in many cases there is a lack of flexibility in sharing the energy resources amongst the multiple transmitting antennas, since it is common to have individual per-antenna amplifiers. This justifies the need to consider power limitations independently for each transmitter. In addition to this, another typical challenge in multi-antenna wireless communication systems is the need to control the instantaneous variations of the per-antenna transmit power. This is important since the power amplifiers fed by the transmitted waveforms usually introduce non-linear effects [19], hence good dynamic properties are required in order to limit the distortion effects. A usual way to deal with this problem in single-user links relies on predistortion techniques [20]. However, their extension to multi-user systems relying on precoding is not straightforward, due to the complex nature of the constellations produced by the precoding operation.

The proposed symbol-level precoding design allows to control the instantaneous per-antenna transmit power, thus leading to a reduction of the power peaks, which are detrimental with respect to the aforementioned non-linearity problem. It should be mentioned that this is not possible in the channellevel approach, where the precoder is designed for an entire codeword, including several symbols, hence the transmitted power can be controlled only in average and not symbol by symbol. As a consequence, the precoded waveforms can show bad dynamic properties in terms of power peaks [21].

The remainder of the paper is organized as follows. In Section 2, the system and signals communication model is delineated. In Section 3, the problem of CI for per-antenna power minimization is proposed and solved. In Section 4, the proposed approach is validated through simulation results. Finally, in Section 5 conclusions are drawn.

Notation: We use upper-case and lower-case bold-faced letters to denote matrices and vectors, respectively. $(\cdot)^{T}$ denotes the transpose of $(\cdot) .|\cdot|$ and $\angle(\cdot)$ denote the amplitude and the phase of $(\cdot)$, respectively, while $\operatorname{Re}(\cdot)$ and $\operatorname{Im}(\cdot)$ are the real and imaginary parts of $(\cdot)$. $\|\cdot\|$ and $\|\cdot\|_{\infty}$ represent the Euclidean norm and the $1_{\infty}$ norm of $(\cdot)$, respectively. Finally, $\operatorname{diag}(\cdot)$ denotes a diagonal matrix whose diagonal entries are the elements of $(\cdot)$, while $\circ$ is used for denoting the elementwise Hadamard operations.

\section{System And Signals Model}

We consider a single-cell multiple-antenna downlink scenario, where a single base-station (BS) is equipped with $N_{t}$ transmit antennas that serve $K$ user terminals, with $K \leq N_{t}$, each one equipped with a single receiving antenna. The adopted modulation is M-PSK, and a block fading channel $\boldsymbol{h}_{j} \in \mathbb{C}^{1 \times N_{t}}$ is assumed between the transmit BS antennas and the $j$-th user. The received signal at the $j$-th user in the symbol slot $n$ can be written as:

$$
y_{j}[n]=\boldsymbol{h}_{j} \boldsymbol{x}[n]+z_{j}[n],
$$

where $\boldsymbol{x}[n] \in \mathbb{C}^{N_{t} \times 1}$ represents the transmitted signal vector from the $N_{t}$ transmit antennas, and $z_{j}[n]$ is a complex circular symmetric random variable, modeling the zero mean Additive White Gaussian Noise (AWGN) measured at the $j$-th user's receiving antenna. Without loss of generality, the noise variance is assumed to be 1 .

By collecting the received signals by all the users in a vector $\boldsymbol{y}[n] \in \mathbb{C}^{K \times 1}$, the above model can be rewritten in a compact form as:

$$
\boldsymbol{y}[n]=\boldsymbol{H} \boldsymbol{x}[n]+\boldsymbol{z}[n],
$$

where $\boldsymbol{H}=\left[\boldsymbol{h}_{1}^{T} \ldots \boldsymbol{h}_{K}^{T}\right]^{T} \in \mathbb{C}^{K \times N_{t}}$ represents the system channel matrix, and $\boldsymbol{z}[n] \in \mathbb{C}^{K \times 1}$ collects the AWGN components for all the users.

According to the symbol-level precoding approach [14], the transmitted signal vector $\boldsymbol{x}[n]$ is obtained as output of a precoding module, which directly designs $\boldsymbol{x}[n]$ using the CSI, which is an estimate of $\boldsymbol{H}$, and the input data symbols $\boldsymbol{d}[n] \in \mathbb{C}^{K \times 1}$, namely the DI that the BS wants to convey to the users. The data symbols, drawn from an M-PSK constellation, are assumed to be uncorrelated and having unit power.

\section{Constructive Interference for PeAK Power MINIMIZATION (CIPPM)}

Following the definition of constructive interference provided in [14], the objective is to design the transmitted vector $\boldsymbol{x}$ (to ease the notation, hereafter the time index $n$ is omitted), based on the CSI and the DI, assuring that the received signal lies in the detection region of the desired symbol, for each user. In other words, we should force the interfering signals to constructively contribute to the useful received power. Moreover, unlike [14], where the total transmit power is minimized, we aim to minimize the per-antenna transmit power. To this end, the proposed approach is to minimize the maximum power among the different transmitters. Such minimization can be seen as the minimization of the peak power between the antennas, thus the proposed scheme is referred to as constructive interference for peak power minimization (CIPPM). The resulting optimization problem can be written as:

$$
\begin{aligned}
\boldsymbol{x}(\boldsymbol{d}, \boldsymbol{H}, \boldsymbol{\gamma})= & \arg \min _{\boldsymbol{x}} \max _{i=1, \ldots, N_{t}}\left\{\left|x_{i}\right|^{2}\right\} \\
& \text { subject to } \\
& \left|\boldsymbol{h}_{j} \boldsymbol{x}\right|^{2} \geq \gamma_{j}, j=1, \ldots, K, \\
& \angle \boldsymbol{h}_{j} \boldsymbol{x}=\angle d_{j}, j=1, \ldots, K,
\end{aligned}
$$

where $\gamma_{j}$ is the target SINR that should be granted for the $j$-th user, and $\gamma=\left[\gamma_{1} \ldots \gamma_{K}\right]^{T} \in \mathbb{C}^{K \times 1}$ contains the target SINR for all the users. The first set of constraints is a QoS constraint for each user. The second set of constraints represents the constructive interference condition, guaranteeing that each user receives the desired data symbol. 
Following the method of [22], we carry out the following steps in order to write the problem (3) in a more tractable form. The equality constraint in (3) can be rewritten, by applying the tangent operator ${ }^{1}$, as:

$$
\frac{\operatorname{Im}\left(\boldsymbol{h}_{j} \boldsymbol{x}\right)}{\operatorname{Re}\left(\boldsymbol{h}_{j} \boldsymbol{x}\right)}=\alpha_{j}, j=1, \ldots, K,
$$

where $\alpha_{j}=\tan \left(\angle d_{j}\right)$. However, since the tangent is not a one-to-one function, the following constraints should be added, in order to ensure that the received symbol and the intended one lie in the same quadrant:

$$
\begin{aligned}
& \operatorname{Re}\left(d_{j}\right) \operatorname{Re}\left(\boldsymbol{h}_{j} \boldsymbol{x}\right) \geq 0, j=1, \ldots, K, \\
& \operatorname{Im}\left(d_{j}\right) \operatorname{Im}\left(\boldsymbol{h}_{j} \boldsymbol{x}\right) \geq 0, j=1, \ldots, K .
\end{aligned}
$$

Concerning the inequality constraint in the problem (3), it can be rewritten referring to the amplitude levels of the in-phase and quadrature components of the corresponding symbols, as follows:

$$
\begin{aligned}
& \left|\operatorname{Re}\left(\boldsymbol{h}_{j} \boldsymbol{x}\right)\right| \geq \sqrt{\gamma_{j}}\left|\operatorname{Re}\left(d_{j}\right)\right|, j=1, \ldots, K, \\
& \left|\operatorname{Im}\left(\boldsymbol{h}_{j} \boldsymbol{x}\right)\right| \geq \sqrt{\gamma_{j}}\left|\operatorname{Im}\left(d_{j}\right)\right|, j=1, \ldots, K,
\end{aligned}
$$

where the absolute value is necessary for accounting negative components. By multiplying both the members of the above equations by $\operatorname{Re}\left(d_{j}\right)$ and $\operatorname{Im}\left(d_{j}\right)$ respectively, the above conditions become:

$$
\begin{aligned}
& \operatorname{Re}\left(d_{j}\right) \operatorname{Re}\left(\boldsymbol{h}_{j} \boldsymbol{x}\right) \geq \sqrt{\gamma_{j}} \operatorname{Re}^{2}\left(d_{j}\right), j=1, \ldots, K, \\
& \operatorname{Im}\left(d_{j}\right) \operatorname{Im}\left(\boldsymbol{h}_{j} \boldsymbol{x}\right) \geq \sqrt{\gamma_{j}} \operatorname{Im}^{2}\left(d_{j}\right), j=1, \ldots, K .
\end{aligned}
$$

It is worth noticing that the constraints in (7) include the ones shown in (5).

Modeling the constraints as shown in (4) and (7), and resorting to the concept of $1_{\infty}$ norm, the CIPPM problem can be rewritten as:

$$
\begin{aligned}
\boldsymbol{x}(\boldsymbol{d}, \boldsymbol{H}, \boldsymbol{\gamma}) & =\arg \min _{\boldsymbol{x}}\|\boldsymbol{x}\|_{\infty} \\
& \text { subject to } \\
& \operatorname{Re}\left(d_{j}\right) \operatorname{Re}\left(\boldsymbol{h}_{j} \boldsymbol{x}\right) \geq \sqrt{\gamma_{j}} \operatorname{Re}^{2}\left(d_{j}\right), j=1, \ldots, K, \\
& \operatorname{Im}\left(d_{j}\right) \operatorname{Im}\left(\boldsymbol{h}_{j} \boldsymbol{x}\right) \geq \sqrt{\gamma_{j}} \operatorname{Im}^{2}\left(d_{j}\right), j=1, \ldots, K, \\
& \frac{\operatorname{Im}\left(\boldsymbol{h}_{j} \boldsymbol{x}\right)}{\operatorname{Re}\left(\boldsymbol{h}_{j} \boldsymbol{x}\right)}=\alpha_{j}, j=1, \ldots, K .
\end{aligned}
$$

Ultimately, the problem can be rewritten in a more compact form as:

$$
\begin{aligned}
\boldsymbol{x}(\boldsymbol{d}, \boldsymbol{H}, \boldsymbol{\gamma})= & \arg \min _{\boldsymbol{x}}\|\boldsymbol{x}\|_{\infty} \\
& \text { subject to } \\
& \operatorname{Re}(\boldsymbol{D}) \operatorname{Re}(\boldsymbol{H} \boldsymbol{x}) \geq \boldsymbol{\beta}_{\boldsymbol{r}} \\
& \operatorname{Im}(\boldsymbol{D}) \operatorname{Im}(\boldsymbol{H} \boldsymbol{x}) \geq \boldsymbol{\beta}_{\boldsymbol{i}} \\
& \boldsymbol{A} \operatorname{Re}(\boldsymbol{H} \boldsymbol{x})-\operatorname{Im}(\boldsymbol{H} \boldsymbol{x})=\mathbf{0},
\end{aligned}
$$

\footnotetext{
${ }^{1}$ This does not apply for data symbols laying on the imaginary axis, since the tangent is not defined in such case. Although this case can be easily handled, it is not considered herein, since we can always assume a phase offset preventing this situation.
}

where $\boldsymbol{D}=\operatorname{diag}(\boldsymbol{d}), \boldsymbol{A}=\operatorname{diag}\left(\alpha_{1}, \ldots, \alpha_{K}\right), \boldsymbol{\beta}_{\boldsymbol{r}}=\sqrt{\boldsymbol{\gamma}} \circ$ $\operatorname{Re}(\boldsymbol{d})^{\circ 2}, \boldsymbol{\beta}_{\boldsymbol{i}}=\sqrt{\boldsymbol{\gamma}} \circ \operatorname{Im}(\boldsymbol{d})^{\circ 2}$.

A way to tackle the problem (9) is to write it as a secondorder cone programming (SOCP) [23] in the stacked variable $\tilde{\boldsymbol{x}}=\left[\operatorname{Re}(\boldsymbol{x})^{T}, \operatorname{Im}(\boldsymbol{x})^{T}\right]^{T} \in \mathbb{R}^{2 N_{t} \times 1}$. To this end, the objective function, as well as the constraints, should be written in terms of $\tilde{\boldsymbol{x}}$.

Concerning the objective function, it is not difficult to see that:

$$
\|\boldsymbol{x}\|_{\infty}=\max _{i=1, \ldots, N_{t}}\left\{\left|x_{i}\right|\right\}=\max _{i=1, \ldots, N_{t}}\left\|\boldsymbol{B}_{i} \tilde{\boldsymbol{x}}\right\|,
$$

where $\boldsymbol{B}_{i} \in \mathbb{R}^{2 \times 2 N_{t}}$ is a matrix used for selecting $\operatorname{Re}\left(x_{i}\right)$ and $\operatorname{Im}\left(x_{i}\right)$ in the stacked vector $\tilde{\boldsymbol{x}}$ and, $\forall i=1, \ldots, N_{t}$, is defined as:

$$
\left[\begin{array}{cc}
\boldsymbol{e}_{i} & \mathbf{0}_{N_{t}} \\
\mathbf{0}_{N_{t}} & \boldsymbol{e}_{i}
\end{array}\right]
$$

with $e_{i}$ being a the $i$-th row of an identity matrix with size $N_{t}$, and $\mathbf{0}_{N_{t}}$ being the all zero entries vector in $\mathbb{R}^{1 \times N_{t}}$.

For writing also the constraints of (9) in terms of $\tilde{\boldsymbol{x}}$, it is convenient to split the vector $\boldsymbol{H} \boldsymbol{x}$ into its real and imaginary parts:

$$
\begin{aligned}
\boldsymbol{H} \boldsymbol{x}= & \operatorname{Re}(\boldsymbol{H}) \operatorname{Re}(\boldsymbol{x})-\operatorname{Im}(\boldsymbol{H}) \operatorname{Im}(\boldsymbol{x})+ \\
& +i[\operatorname{Re}(\boldsymbol{H}) \operatorname{Im}(\boldsymbol{x})+\operatorname{Im}(\boldsymbol{H}) \operatorname{Re}(\boldsymbol{x})],
\end{aligned}
$$

which leads straightforwardly to:

$$
\operatorname{Re}(\boldsymbol{H} \boldsymbol{x})=\boldsymbol{H}_{\mathbf{1}} \tilde{\boldsymbol{x}}, \quad \operatorname{Im}(\boldsymbol{H} \boldsymbol{x})=\boldsymbol{H}_{\mathbf{2}} \tilde{\boldsymbol{x}},
$$

where $\boldsymbol{H}_{\mathbf{1}}=[\operatorname{Re}(\boldsymbol{H}),-\operatorname{Im}(\boldsymbol{H})], \boldsymbol{H}_{\mathbf{2}}=[\operatorname{Im}(\boldsymbol{H}), \operatorname{Re}(\boldsymbol{H})]$. Hence, the optimization problem (9) becomes:

$$
\begin{aligned}
\tilde{\boldsymbol{x}}(\boldsymbol{d}, \boldsymbol{H}, \gamma)= & \arg \min _{\tilde{\boldsymbol{x}}} \max _{i=1, \ldots, N_{t}}\left\|\boldsymbol{B}_{i} \tilde{\boldsymbol{x}}\right\| \\
& \text { subject to } \\
& \operatorname{Re}(\boldsymbol{D}) \boldsymbol{H}_{\mathbf{1}} \tilde{\boldsymbol{x}} \geq \boldsymbol{\beta}_{\boldsymbol{r}}, \\
& \operatorname{Im}(\boldsymbol{D}) \boldsymbol{H}_{\mathbf{2}} \tilde{\boldsymbol{x}} \geq \boldsymbol{\beta}_{\boldsymbol{i}}, \\
& \left(\boldsymbol{A} \boldsymbol{H}_{\mathbf{1}}-\boldsymbol{H}_{\mathbf{2}}\right) \tilde{\boldsymbol{x}}=\mathbf{0} .
\end{aligned}
$$

Finally, by introducing a slack variable $r$, the CIPPM problem can be formulated as a SOCP as follows:

$$
\begin{aligned}
\tilde{\boldsymbol{x}}(\boldsymbol{d}, \boldsymbol{H}, \boldsymbol{\gamma})= & \arg \min _{r, \tilde{\boldsymbol{x}}} r \\
& \text { subject to } \\
& \left\|\boldsymbol{B}_{i} \tilde{\boldsymbol{x}}\right\| \leq r, i=1, \ldots, N_{t}, \\
& \operatorname{Re}(\boldsymbol{D}) \boldsymbol{H}_{\mathbf{1}} \tilde{\boldsymbol{x}} \geq \boldsymbol{\beta}_{\boldsymbol{r}} \\
& \operatorname{Im}(\boldsymbol{D}) \boldsymbol{H}_{\mathbf{2}} \tilde{\boldsymbol{x}} \geq \boldsymbol{\beta}_{\boldsymbol{i}} \\
& \left(\boldsymbol{A} \boldsymbol{H}_{\mathbf{1}}-\boldsymbol{H}_{\mathbf{2}}\right) \tilde{\boldsymbol{x}}=\mathbf{0} .
\end{aligned}
$$

The global optimum of this optimization problem can be obtained using the standard convex optimization tools. 


\section{Simulation Results}

In this section some numerical results are presented, to show the effectiveness of the proposed CIPPM approach. Before discussing the results, let us define the considered performance metrics. The symbol-level average power transmitted by each antenna is defined as $P_{\mathrm{av}}=\frac{\|\boldsymbol{x}\|^{2}}{N_{t}}$, whilst the symbol-level peak power among the antennas will be $P_{\text {peak }}=\|\boldsymbol{x}\|_{\infty}^{2}$. By taking an average of such quantities over a large number of symbol slots, we obtain the frame-level average power and peak power, which are used as performance metric hereafter. Furthermore, the peak-to-average power ratio (PAPR), intended as the ratio of the introduced power metrics, is also considered to quantify the relative weight of the power peaks.

The numerical results of the proposed scheme are compared with the ones obtained with the approach of [14], which we can refer to as constructive interference for sum power minimization (CISPM). The corresponding optimization problem is the following:

$$
\begin{aligned}
\boldsymbol{x}(\boldsymbol{d}, \boldsymbol{H}, \boldsymbol{\gamma})= & \arg \min _{\boldsymbol{x}}\|\boldsymbol{x}\|^{2} \\
& \text { subject to } \\
& \left|\boldsymbol{h}_{j} \boldsymbol{x}\right|^{2} \geq \gamma_{j}, j=1, \ldots, K, \\
& \angle \boldsymbol{h}_{j} \boldsymbol{x}=\angle d_{j}, j=1, \ldots, K .
\end{aligned}
$$

The presented results in Figs. 1-4 have been obtained by averaging over 500 frames of $N=100$ symbol slots each. The quasi-static block fading channel coefficients have been generated, for the generic user $j$, as $\boldsymbol{h}_{j} \sim \mathcal{C N}\left(0, \sigma_{h}^{2} \boldsymbol{I}\right)$, with $\sigma_{h}^{2}=1$. Each fading block is assumed to correspond to a frame. Moreover, the assumed modulation scheme is QPSK. Finally, the number of transmit antennas $N_{t}$ is assumed to be equal to the number of users $K$, and such number will be hereafter referred to as system size.

Fig. 1 shows the introduced power consumption metrics, in $\mathrm{dBW}$, as a function of the target SINR, assumed the same for all the users for the sake of simplicity. The system size is fixed to 10. As expected, it can be seen how the required transmit power increases with the target SINR, and how the proposed CIPPM approach attains better performance in terms of peak power with respect to the CISPM approach. This gain on the peak power, close to $1 \mathrm{~dB}$, comes with the sacrifice of a higher average transmit power. As a result, we have a lower PAPR (3.1 $\mathrm{dB})$ with the proposed CIPPM approach, with respect to the CISPM one $(4.7 \mathrm{~dB})$.

Fig. 2 shows the power consumption, in $\mathrm{dBW}$, as a function of the system size, for a target SINR fixed to $3 \mathrm{~dB}$. Besides the fact that the CIPPM approach outperforms the CISPM one in terms of peak power, it is worth noticing how the power has a decreasing trend with the system size. This can be intuitively explained considering that, for a higher numbers of transmitting antennas, the stronger effect of constructive interference allows to achieve the target SINR with a lower required power. Moreover, it is important to notice how the performance gap between the two compared techniques increases with the system size. This implies that much higher

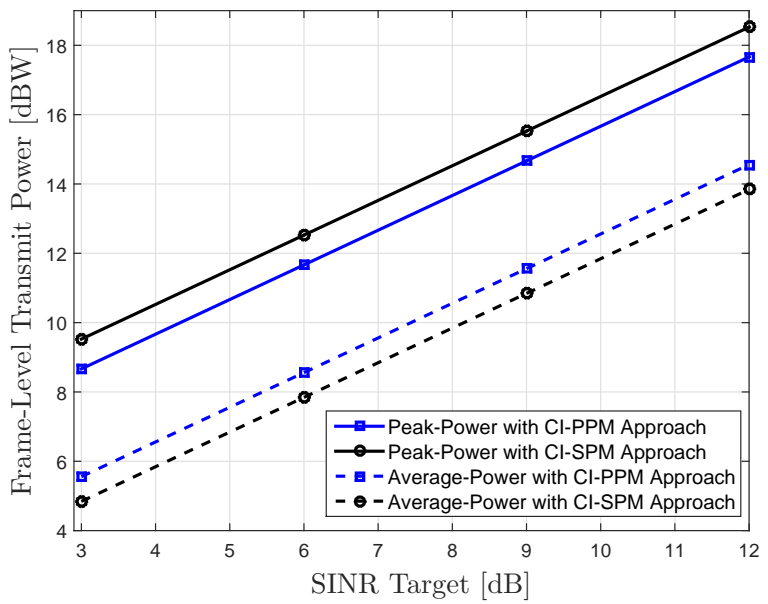

Figure 1: Frame-level transmit power in dBW vs. SINR target in dB.

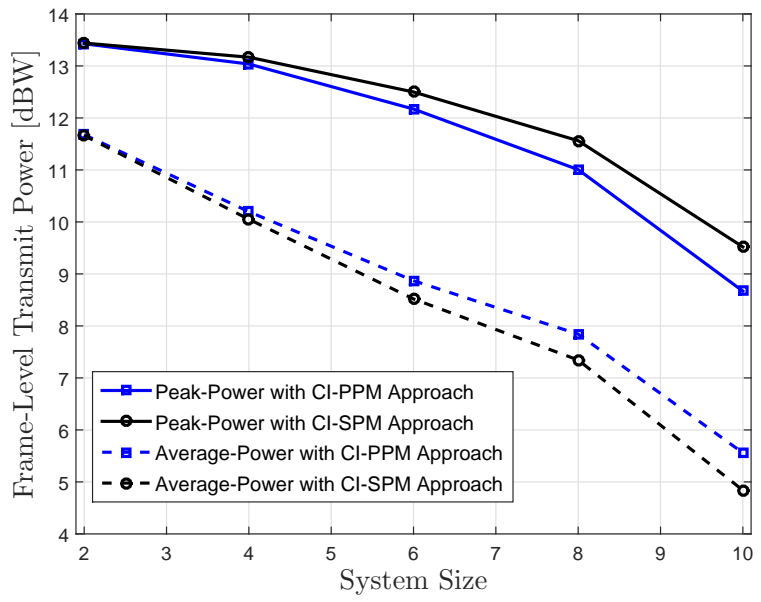

Figure 2: Frame-level transmit power in dBW vs. system size.

gains can be expected for very high system size value. This is the case of large antenna arrays in massive MIMO [24] and of multi-beam satellite systems [21]. Further insights can be given by the PAPR curves shown in Fig. 3. In fact, with the proposed approach the PAPR is remarkably lower when the system size increases.

Fig. 4 shows the power consumption as a function of the effective user rate, assuming the system size fixed to 10 . The effective user rate is clearly related to the target SINR and, for a generic user $j$, can be defined as:

$$
\bar{R}_{j}=R_{j}(1-\mathrm{SER}),
$$

where $R_{j}$ denotes the maximum rate, in bits per symbol, supported by the adopted modulation, and SER is the symbol error rate. It can be seen how the maximum supported user rate of $2 \mathrm{bits} / \mathrm{s} / \mathrm{Hz}$ is attained with a lower peak power with the proposed approach.

Finally, Fig. 5 shows the instantaneous power utilization, in linear scale, for each transmit antenna, with the CIPPM and 


$$
\begin{gathered}
\boldsymbol{H}=\left[\begin{array}{ccccc}
-0.1233+0.5238 i & 0.7062+0.2725 i & 0.8247-0.1884 i & -1.4108-0.5979 i & -1.3513+0.9245 i \\
-0.7921-0.6147 i & 0.2095+0.5430 i & 0.6066+1.8920 i & -0.2581-0.5440 i & -0.7350-0.7109 i \\
0.7792-1.3379 i & -0.5975+1.0688 i & 0.7686+0.9474 i & -0.5657-1.0041 i & 0.2530+0.5123 i \\
0.7827+0.0125 i & 0.3131+0.4758 i & -0.3386+0.2376 i & -0.8578+1.2915 i & 0.0653+0.0528 i \\
-0.3473-1.0009 i & 0.6973-0.2266 i & -0.1827+0.5567 i & 1.3305-0.4221 i & -0.1453-0.2645 i
\end{array}\right], \\
\boldsymbol{d}=\left[\begin{array}{lllll}
0.7071+0.7071 i & 0.7071+0.7071 i & 0.7071+0.7071 i & -0.7071+0.7071 i & -0.7071-0.7071 i
\end{array}\right]^{T}
\end{gathered}
$$

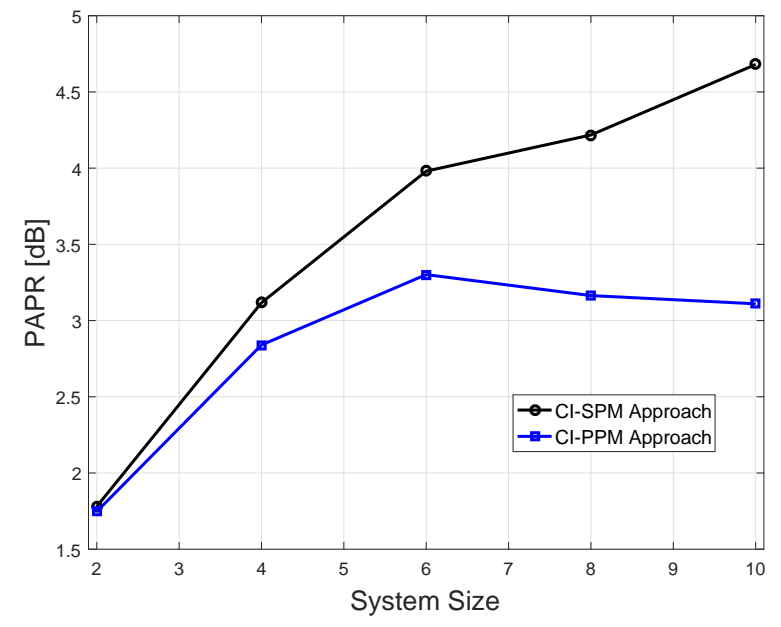

Figure 3: PAPR in $\mathrm{dB}$ vs. system size.

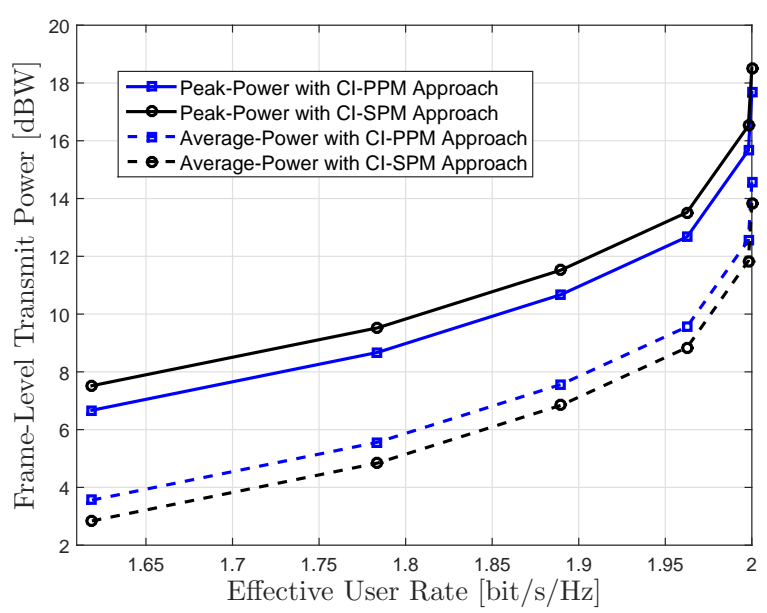

Figure 4: Frame-level transmit power in $\mathrm{dBW}$ vs. effective user rate in $\mathrm{bit} / \mathrm{s} / \mathrm{Hz}$.

the CISPM approaches, for a $5 \times 5$ system and a target SINR of $3 \mathrm{~dB}$. The channel matrix and the DI vector are fixed to the values reported in (17), (18). Such representation clearly shows how, sacrificing some average power, the proposed approach leads to a more uniform distribution of the power between the antennas, resulting in a lower peak power.

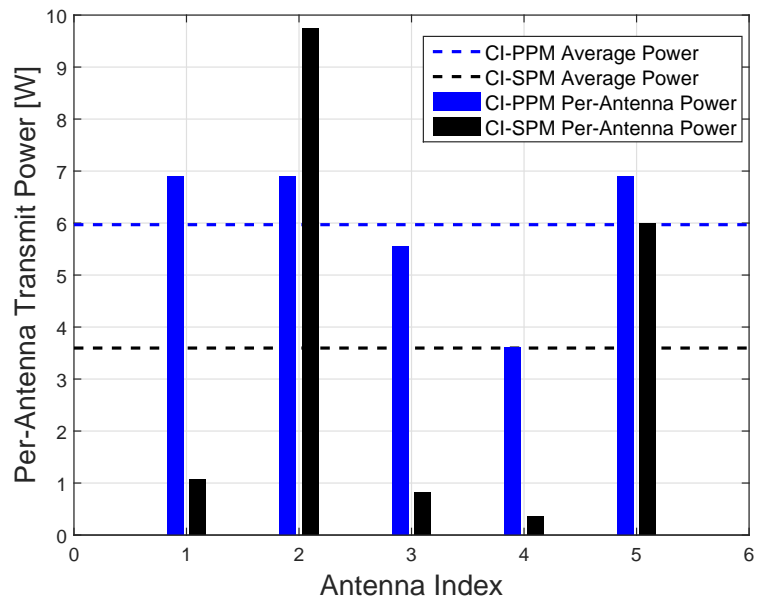

Figure 5: Per-antenna power utilization in Watts for a $5 \times 5$ system.

\section{CONCLUSIONS}

In the present work, a novel approach for symbol-level precoding is proposed, aiming at minimizing the per-antenna transmit power. In particular, the problem of minimization of the peak power amongst the transmitting antenna, under QoS constraints, is formulated and solved, in order to have a more uniform distribution of the transmitted power with respect to the state of the art symbol-level techniques. Such design is particularly suitable for per-antenna power limited systems, as well as for systems corrupted by non-linear effects. The performance of the proposed scheme is assessed through numerical results, in comparison to the sum power minimization scheme. In particular, it is shown how the proposed approach gains in terms of a reduced transmitted peak power across the antennas, at the expense of a higher required average power.

\section{ACKNOWLEDGMENT}

This work was partially supported by the National Research Fund, Luxembourg, under the projects SATSENT, SeMiGod, and BroadSat (AFR project).

\section{REFERENCES}

[1] R. Roy and B. Ottersten, "Spatial division multiple access wireless communication systems," June 1997, US Patent 5,642,353. [Online]. Available: https://www.google.com/patents/US5642353

[2] Y.-F. Liu, Y.-H. Dai, and Z.-Q. Luo, "Coordinated beamforming for MISO interference channel: Complexity analysis and efficient algorithms," IEEE Transactions on Signal Processing, vol. 59, no. 3, pp. 1142-1157, 2011. 
[3] E. Björnson, M. Bengtsson, and B. Ottersten, "Optimal multiuser transmit beamforming: A difficult problem with a simple solution structure [lecture notes]," IEEE Signal Processing Magazine, vol. 31, no. 4, pp. 142-148, July 2014.

[4] A. B. Gershman, N. D. Sidiropoulos, S. Shahbazpanahi, M. Bengtsson, and B. Ottersten, "Convex optimization-based beamforming," IEEE Signal Processing Magazine, vol. 27, no. 3, pp. 62-75, May 2010.

[5] M. Bengtsson and B. Ottersten, "Optimal and suboptimal transmit beamforming," in Handbook of Antennas in Wireless Communications. CRC Press, 2001, pp. 18-1-18-33, qC 20111107.

[6] M. Schubert and H. Boche, "Solution of the multiuser downlink beamforming problem with individual sinr constraints," IEEE Transactions on Vehicular Technology, vol. 53, no. 1, pp. 18-28, Jan 2004.

[7] M. Bengtsson and B. Ottersten, "Optimal downlink beamforming using semidefinite optimization," in Proc. of Annual Allert. Conf. on Commun. Control and Computing, vol. 37. Citeseer, 1999, pp. 987-996.

[8] W. Yu and T. Lan, "Transmitter optimization for the multi-antenna downlink with per-antenna power constraints," IEEE Transactions on Signal Processing, vol. 55, no. 6, pp. 2646-2660, June 2007.

[9] G. Dartmann, X. Gong, W. Afzal, and G. Ascheid, "On the duality of the max min beamforming problem with per-antenna and per-antennaarray power constraints," IEEE Transactions on Vehicular Technology, vol. 62, no. 2, pp. 606-619, Feb 2013.

[10] D. Christopoulos, S. Chatzinotas, and B. Ottersten, "Weighted fair multicast multigroup beamforming under per-antenna power constraints," IEEE Transactions on Signal Processing, vol. 62, no. 19, pp. 51325142, Oct 2014.

[11] C. Masouros and E. Alsusa, "Dynamic linear precoding for the exploitation of known interference in mimo broadcast systems," IEEE Transactions on Wireless Communications, vol. 8, no. 3, pp. 1396-1404, March 2009.

[12] C. Masouros, "Correlation rotation linear precoding for mimo broadcast communications," IEEE Transactions on Signal Processing, vol. 59, no. 1 , pp. $252-262$, Jan. 2011

[13] M. Alodeh, S. Chatzinotas, and B. Ottersten, "A multicast approach for constructive interference precoding in miso downlink channel," in Information Theory (ISIT), 2014 IEEE International Symposium on, June 2014, pp. 2534-2538.

[14] M. Alodeh, S. Chatzinotas, and B. Ottersten, "Constructive multiuser interference in symbol level precoding for the miso downlink channel," IEEE Transactions on Signal Processing,, vol. 63, no. 9, pp. 2239-2252, May 2015.

[15] M. Alodeh, S. Chatzinotas, and B. Ottersten, "Constructive interference through symbol level precoding for multi-level modulation," in 2015 IEEE Global Communications Conference (GLOBECOM), Dec 2015, pp. 1-6.

[16] M. Alodeh, S. Chatzinotas, and B. Ottersten, "Energy efficient symbollevel precoding in multiuser miso channels," in Signal Processing Advances in Wireless Communications (SPAWC), 2015 IEEE 16th International Workshop on, June 2015, pp. 36-40.

[17] M. Alodeh, S. Chatzinotas, and B. Ottersten, "Energy-efficient symbollevel precoding in multiuser miso based on relaxed detection region," IEEE Transactions on Wireless Communications,, vol. PP, no. 99, pp. 1-1, 2016.

[18] C. Masouros and G. Zheng, "Exploiting known interference as green signal power for downlink beamforming optimization," IEEE Transactions on Signal Processing, vol. 63, no. 14, pp. 3628-3640, July 2015.

[19] G. Auer, V. Giannini, C. Desset, I. Godor, P. Skillermark, M. Olsson, M. A. Imran, D. Sabella, M. J. Gonzalez, O. Blume, and A. Fehske, "How much energy is needed to run a wireless network?" IEEE Wireless Communications, vol. 18, no. 5, pp. 40-49, October 2011.

[20] R. Piazza, M. R. B. Shankar, and B. Ottersten, "Data predistortion for multicarrier satellite channels based on direct learning," IEEE Transactions on Signal Processing, vol. 62, no. 22, pp. 5868-5880, Nov 2014.

[21] D. Spano, D. Christopoulos, S. Andrenacci, S. Chatzinotas, J. Krause, and B. Ottersten, "Total degradation analysis of precoded signals onto non-linear satellite channels," in 21st Ka and Broadband Communications Conference, Oct 2015.

[22] A. Kalantari, M. Soltanalian, S. Maleki, S. Chatzinotas, and B. Ottersten, "Secure m-psk communication via directional modulation," in Acoustics, Speech, and Signal Processing, 2016. Proceedings. (ICASSP '16). IEEE International Conference on, March 2016.
[23] S. Boyd and L. Vandenberghe, Convex optimization. Cambridge Univ. Press, 2004

[24] E. Larsson, O. Edfors, F. Tufvesson, and T. Marzetta, "Massive mimo for next generation wireless systems," IEEE Communications Magazine, vol. 52, no. 2, pp. 186-195, February 2014. 